\title{
Variabilidad espacial en la estructura de la ictiofauna asociada a praderas de Cymodocea nodosa en las Islas Canarias, Atlántico nororiental subtropical
}

\author{
Spatial variability in the structure of the ichthyofauna associated with Cymodocea nodosa \\ seagrass meadows across the Canary Islands, north-eastern subtropical Atlantic
}

\author{
Fernando Espino ${ }^{1}$, Fernando Tuya ${ }^{1}$, Alberto Brito ${ }^{2}$ y Ricardo Haroun ${ }^{1}$
}

\begin{abstract}
${ }^{1}$ Centro de Investigación en Biodiversidad y Gestión Ambiental (BIOGES), Facultad de Ciencias del Mar, Campus de Tafira, Universidad de Las Palmas de Gran Canaria, 35017 Las Palmas, Gran Canaria, Islas Canarias, España. fesprod@gobiernodecanarias.org

${ }^{2}$ Grupo de Investigación BIOECOMAC, Unidad de Ciencias Marinas, Departamento de Biología Animal, Facultad de Biología, Universidad de La Laguna, 38206, La Laguna, Tenerife, Islas Canarias, España
\end{abstract}

\begin{abstract}
A description of the patterns in spatial variability of fish assemblages is particularly relevant to guarantee an adequate management of these marine resources. Thirty six trawls were considered on six seagrass meadows (10 km apart) in three islands (100 km apart) of the Canary Islands to (i) analyze the spatial variability in the structure (richness, abundance and diversity) of fish assemblages associated with Cymodocea nodosa seagrass meadows, and to (ii) assess how the structural complexity of this habitat affects fish assemblage structure. A total of 3,616 fishes were captured, belonging to 30 species and 15 families. The total length of ca. $95 \%$ of individuals was $<10 \mathrm{~cm}$, and ca. $90 \%$ of individuals were juveniles. Spatial variability in fish assemblage structure was larger when considering the scale of meadows than the scale of islands $(27.28 \%$ vs. $6.64 \%$ respectively, in the amount of explained total variability); the smallest spatial scale (i.e., the variability among replicated trawls within each seagrass meadow) accumulated the largest amount of variability (66.07\%). Differences in fish assemblage structure were largely driven by changes in the abundance of a few species, including Diplodus annularis, Spondyliosoma cantharus, Mullus surmuletus and Symphodus trutta. These species recruited into seagrass meadows, and confirmed the nursery role of this habitat in the canarian coastal waters. Mean shoot density and variation of the seagrass canopy height were the descriptors that accounted for the most variability when explaining patterns in fish assemblage structure.
\end{abstract}

Key words: Abundance, juvenile fish, nursery, habitat structure, seagrass meadows

Resumen.- El conocimiento de los patrones de variabilidad espacial de las comunidades de peces es de especial relevancia para una adecuada gestión de estos recursos marinos. Con el objetivo de: (i) analizar la variabilidad espacial en la estructura (riqueza, abundancia y diversidad) de la ictiofauna asociada a praderas marinas de Cymodocea nodosa en el Archipiélago Canario y (ii) conocer cómo la complejidad estructural de este hábitat afecta a la comunidad de peces, se realizaron 36 arrastres en seis praderas (separadas por $10 \mathrm{~km}$ ) en tres islas (separadas por $100 \mathrm{~km}$ ). Se registraron un total de 3.616 individuos pertenecientes a 30 especies y 15 familias. La longitud total de ca. $95 \%$ de los individuos fue $<10 \mathrm{~cm}$ y ca. $90 \%$ fueron juveniles. La variabilidad espacial en la estructura de la comunidad asociada a escala de praderas fue superior que a escala de islas $(27,28 \%$ vs. $6,64 \%$ de la variabilidad total); la menor escala (e.g., variabilidad entre arrastres dentro de cada pradera), acumuló la mayor parte de la variabilidad (66,07\%). Las diferencias en la estructura de la comunidad entre praderas se debieron a cambios en la abundancia de determinadas especies, como Diplodus annularis, Spondyliosoma cantharus, Mullus surmuletus y Symphodus trutta, que usan las praderas como lugares de reclutamiento, confirmando el papel de guardería que juega este hábitat en Canarias. La densidad media de pies y la variación en la altura del estrato foliar fueron los parámetros estructurales que más afectaron la estructura de la comunidad de ictiofauna.

Palabras clave: Abundancia, peces juveniles, guardería, estructura del hábitat, praderas marinas 


\section{INTRODUCCIÓN}

Las praderas de fanerógamas marinas constituyen uno de los ecosistemas marinos más importantes, debido a que participan en los procesos biogeoquímicos litorales: su alta productividad primaria y secundaria (Hemminga $\&$ Duarte 2000) las convierten en una fuente de recursos alimenticios y su complejidad estructural provee de un mayor número de hábitats, lo que permite el desarrollo de comunidades más complejas, con una alta abundancia y diversidad de invertebrados y peces (Beck et al. 2001, Gillanders 2006). Además, estas praderas constituyen un hábitat adecuado para el reclutamiento y cría de numerosas especies de peces (Pollard 1984, Bell \& Pollard 1989). En Canarias, las praderas de Cymodocea nodosa (Ucria) Ascherson también desempeñan múltiples funciones ecológicas (Aguilera et al. 1994, Barberá et al. 2005, Espino et al. 2008, 2011), similares a las que realizan otras especies de fanerógamas marinas en áreas templadas y tropicales, lo que las convierte en un ecosistema clave en el litoral de las islas.

El análisis de la variabilidad espacial de las comunidades de peces asociadas a praderas de fanerógamas marinas ha sido objeto de numerosas investigaciones. Sin embargo, la mayoría de los estudios se han limitado a escalas espaciales pequeñas (10 a $100 \mathrm{~m}$; e.g., 'dentro de parches, entre parches'), mientras que variaciones a escalas medias (de 10 a $100 \mathrm{de} \mathrm{km}$, e.g., a 'escala de paisaje') han sido investigadas en menor medida y sólo más recientemente (Gillanders 2006). A escalas espaciales más grandes (> $100 \mathrm{~km}$, e.g., a 'escala regional y/o biogeográfica'), los trabajos son aún más escasos, tal y como la comparación entre distintos estuarios y regiones (Worthington et al. 1992, Ferrell et al. 1993, Wyda et al. 2002, Moranta et al. 2006) y entre distintas regiones biogeográficas (Weinstein \& Heck 1979, Heck \& Wilson 1987, Costa et al. 2002).

La ictiofauna asociada a las praderas de Cymodocea nodosa ha sido objeto de diversos estudios a lo largo de su área de distribución. En el mar Mediterráneo, se han abarcado diferentes aspectos: estudio de la ictiofauna en praderas mixtas de C. nodosa y Zostera noltii (Guidetti \& Bussotti 2000), variación temporal de la comunidad (ReinaHervás \& Serrano 1987), influencia de los factores ambientales sobre la ocupación de hábitats (Bonaca \& Lipej 2005), comparación de ictiofaunas entre distintos tipos de praderas marinas (Manent \& Abella 2005, VerdiellCubedo et al. 2007), cambios en la comunidad por introducción de especies exóticas (Relini et al. 2000) y efectos de la alteración de la bóveda de la pradera sobre la comunidad (Guidetti \& Bussotti 2002). En las costas atlánticas del suroeste ibérico, los estudios son más escasos y comprenden la caracterización general de la comunidad, así como variaciones a diferentes escalas temporales (Ribeiro et al. 2006). De igual forma, los estudios son escasos en las Islas Canarias y se han restringido a: inventario de especies (Mena et al. 1993), impacto de la construcción de un puerto y estudio del ciclo anual de la comunidad (Tuya et al. 2002b, 2006) y comparación entre diferentes hábitats (Tuya et al. 2005, Polifrone et al. 2006). Sin embargo, la variabilidad espacial de la ictiofauna asociada a praderas de C. nodosa a diferentes escalas no ha sido estudiada, ni tampoco su posible relación con la estructura y organización de dichas praderas. En este sentido, son absolutamente necesarios más datos procedentes de un mayor número de praderas y áreas geográficas con el fin de determinar las posibles variaciones a escalas espaciales grandes (Guidetti \& Bussotti 2000).

Las praderas marinas son uno de los ecosistemas más amenazados del planeta (Orth et al. 2006), con una tasa de desaparición de $100 \mathrm{~km}^{2} \mathrm{yr}^{-1}$ (Waycott et al. 2009). En las Islas Canarias, las praderas de Cymodocea nodosa se encuentran en regresión y también su comunidad asociada, debido a la presión antrópica existente en las áreas litorales (Espino et al. 2008). El estudio de los patrones de variabilidad espacial tiene gran importancia ecológica para entender el efecto de los factores ambientales sobre la distribución y abundancia de organismos. Además, también proveen de información valiosa para la gestión y conservación de los ecosistemas. El objetivo de este trabajo fue analizar la variabilidad espacial en la estructura (riqueza, abundancia y diversidad) de la comunidad de peces asociada a praderas marinas de $C$. nodosa a dos escalas espaciales: entre praderas (separadas por $10 \mathrm{~km}$ ) y entre islas (separadas por $100 \mathrm{~km}$ ) a lo largo del Archipiélago Canario. Estas dos escalas han sido empleadas en otros estudios y se consideran adecuadas para la gestión de praderas marinas (Ferrell et al. 1993). Además, se intentó determinar cómo afecta la estructura física de las praderas a la estructura de la comunidad íctica.

\section{Materiales y MÉTODOS}

\section{ÁREA DE ESTUdio}

Se seleccionaron 6 praderas (separadas por $10 \mathrm{~km}$ ) de la fanerógama marina Cymodocea nodosa en 3 islas (separadas por $100 \mathrm{~km}$ ) del Archipiélago Canario (Fig. 1), a 
Figura 1. Zona de estudio: Islas Canarias orientales (Lanzarote, Fuerteventura y Gran Canaria) con las praderas marinas estudiadas en cada isla / Study area: eastern Canary Islands (Lanzarote, Fuerteventura, and Gran Canaria) including the seagrass meadows studied on each island

Islas y su ubicación/Islands and locations: LZ1 = Playa Blanca (UTM 28R0614232; 3192496); LZ2 = Los Pocillos (UTM 28R0633275; 3200219); FV1 = Jandía (UTM 28R0563535; 3102501); FV2 = La Entallada (UTM 28R0602018; 3122713); GC1 = Veneguera (UTM 28R0421370; 3081050); $\quad$ GC2 = Arinaga (UTM 28R0460944; 3080980)

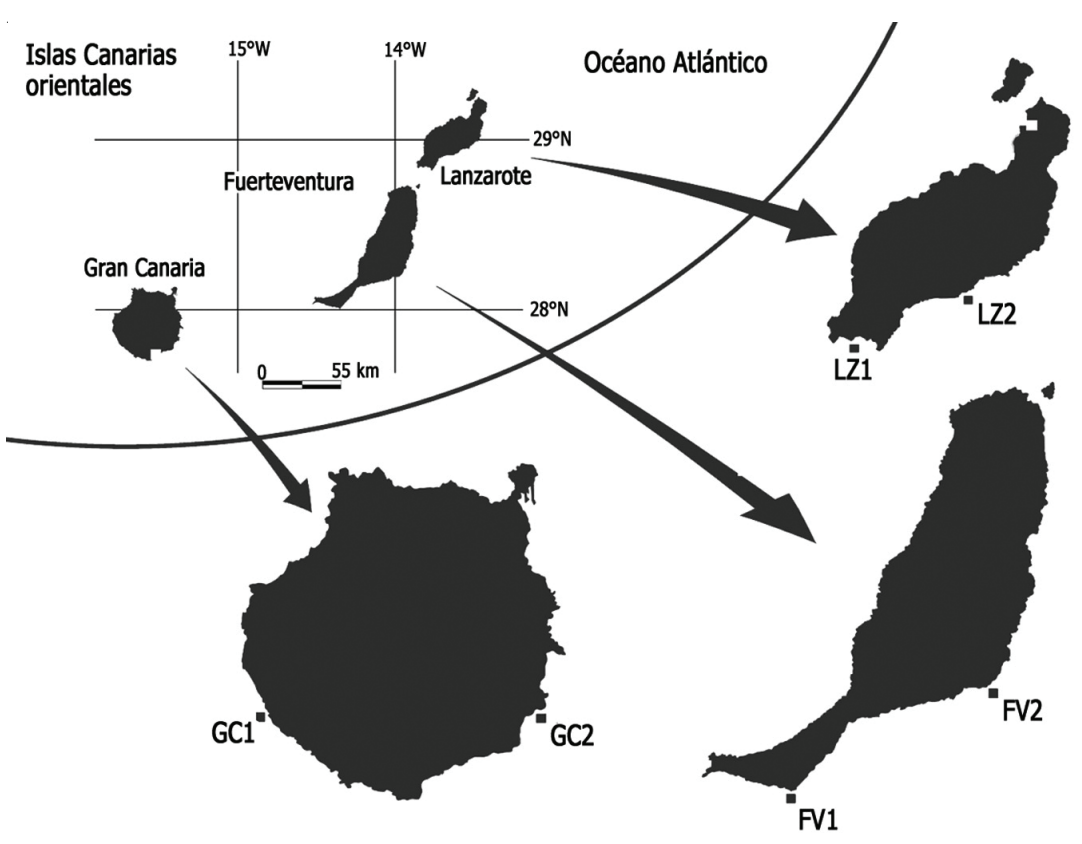

partir de la información disponible sobre la distribución de la especie en Canarias (Reyes et al. 1995a, PavónSalas et al. 2000). Los criterios de selección fueron: i) que la superficie de la pradera marina permitiese realizar los arrastres con una separación de $100 \mathrm{~m}$ y ii) que la distancia mínima entre praderas fuera, al menos, de $10 \mathrm{~km}$. Los vientos alisios procedentes del noreste y la corriente de Canarias determinan las principales estructuras oceanográficas de la región; durante el período primaveraverano, la temperatura media superficial del agua alrededor de las islas Canarias orientales oscila entre $21^{\circ} \mathrm{C}$ y $22,5^{\circ} \mathrm{C}$ y la salinidad entre 36,3 ups y 36,7 ups (Navarro-Pérez \& Barton 2001).

\section{MÉTOdo de MUestreo}

Los muestreos se realizaron mediante una red de arrastre tipo chinchorro de $6 \mathrm{~m}$ de largo, $4 \mathrm{~m}$ de ancho y $0,5 \mathrm{~m}$ de alto, con luz de malla de $1 \mathrm{~mm}$. La red fue arrastrada por buceadores a lo largo de un transecto de $25 \mathrm{~m}$ de longitud, cubriendo un área de $100 \mathrm{~m}^{2}$ por arrastre, con una duración de 5 min cada uno. En cada pradera, se realizaron 6 arrastres al azar y separados, al menos, por $100 \mathrm{~m}$; entre 5 y $15 \mathrm{~m}$ de profundidad y entre las 10:00 y las 15:00 h. Esta técnica fue empleada por Espino et al. (2011) en Canarias y permite la captura de individuos pequeños, con escasa capacidad de natación, que se refugian en el estrato foliar de la pradera; también captura ejemplares de mayor tamaño de especies bentónicas. Las técnicas de arrastre se consideran efectivas en el muestreo de peces pequeños (longitud total $<100 \mathrm{~mm}$ ), (Rozas \& Minello 1997, Guest et al. 2003). Todos los muestreos se efectuaron entre junio y septiembre de 2003, cuando las comunidades de fanerógamas marinas presentan los mayores valores de biomasa en Canarias (Reyes et al. 1995b, Tuya et al. 2006) y diversos descriptores (riqueza y abundancia) de las comunidades de peces asociadas también son máximos (Polifrone et al. 2006, Tuya et al. 2006). Los peces capturados fueron identificados de acuerdo con Fischer et al. $(1981)^{1}$ y Brito et al. (2002) y medidos (longitud total $\pm 1 \mathrm{~mm}$ ). Se clasificaron en juveniles y adultos siguiendo el criterio empleado por Dulcic et al. (1997). Se consideraron juveniles aquellos ejemplares con escamas ya formadas que no han alcanzado la talla de primera madurez sexual mínima (TPMm); los adultos fueron aquellos que alcanzaron y superaron dicha longitud. Para cada especie, esta longitud fue obtenida de las referencias bibliográficas disponibles en Canarias y en determinados casos de otras regiones, (Espino et al. 2011). Para caracterizar la organización espacial de la comunidad se empleó la clasificación utilizada por Ribeiro et al. (2006) para peces en praderas de Cymodocea nodosa, quienes

${ }^{1}$ Fischer W, G Bianchi \& WB Scott. 1981. Eastern Central Atlantic. Fishing Area 34 and part of 47. FAO Species Identification Sheets for Fishery Purposes 1-7: [pag. var.] 
a su vez adaptaron la de Elliott \& Dewailly (1995); aquí, los peces fueron clasificados en bentónicos (que viven sobre o en el substrato) y epibentónicos (que viven en la capa de agua justo sobre el lecho). Para los grupos tróficos, debido a la ausencia de estudios específicos en praderas de $C$. nodosa, se adaptó la empleada por Moranta et al. (2006) en praderas de Posidonia oceanica, donde los peces se clasificaron en: herbívoros, macrófagos, mesófagos, micrófagos y omnívoros. Estas categorías difieren ligeramente de las establecidas por Bell \& Harmelin-Vivien (1983) y Macpherson et al. (2002); y fueron definidas de acuerdo con resultados recientes de niveles tróficos determinados mediante análisis isotópicos (Jennings et al. 1997, Deudero et al. 2004).

Para estimar el efecto de la estructura física de la pradera sobre los patrones de la estructura de la comunidad de peces, se cuantificaron, en cada punto de arrastre, dos elementos estructurales típicamente empleados en estudios sobre comunidades ícticas asociadas a praderas marinas: la densidad de pies y altura de las hojas. Ambos se han mostrado como descriptores eficientes de la complejidad estructural de praderas de Cymodocea nodosa en el Archipiélago Canario (Reyes et al. 1995a, b, Barberá et al.2005). Para estimar la densidad, se contaron los pies de planta en el interior de un cuadrado de 25 x 25 $\mathrm{cm}(\mathrm{n}=10)$, superficie de muestreo empleada en Canarias para estimar la densidad media de pies por Espino (2004) y Barberá et al. (2005); posteriormente, esta densidad fue convertida a $\mathrm{m}^{2}$. Para estimar la altura del estrato foliar, se midió la longitud de las hojas en cada pie $(\mathrm{n}=30)$. La estructura y superficie ocupada por cada pradera fueron calculadas por Espino et al. (2003).

\section{Análisis estadísticos}

Para cada muestra, se calculó la riqueza de especies (S), la abundancia total de individuos, el índice de diversidad de Shannon-Wiener (H'), el índice de Pielou o equidad (J') y la abundancia de cada categoría espacial y trófica. Los patrones de distribución espacial de las especies se describieron mediante el coeficiente de dispersión ( $\mathrm{CD}=$ varianza/media). Se contrastó si cada especie seguía una distribución aleatoria, predicha por una distribución de Poisson, mediante el estadístico $\chi^{2}$, que contrastó las diferencias entre las distribución observada y esperada para cada especie (Ludwig \& Reynolds 1988). Se aplicó un análisis univariante (ANDEVA) para contrastar las diferencias entre islas (factor fijo) y praderas (factor aleatorio), en los descriptores univariantes de la comunidad. Se comprobaron las asunciones de normalidad y homogeneidad de la varianza aplicando, previamente, las pruebas de Kolmogorov-Smirnov y Cochram, respectivamente. Cuando estas suposiciones no se alcanzaron, los datos fueron transformados mediante raíz cuadrada. Para visualizar las diferencias en la estructura de la comunidad íctica entre islas, se ejecutó un análisis canónico de coordenadas principales (ACP), (Anderson \& Willis 2003). Las diferencias en la estructura de la comunidad entre islas (factor fijo) y praderas dentro de cada isla (factor aleatorio), se contrastaron aplicando un análisis multivariante de la varianza (AMDEVA, Anderson 2001), basado en el índice de similitud de BrayCurtis para datos trasformados (raíz cuadrada). La contribución de cada especie a la disimilitud en la estructura de la comunidad íctica entre islas se obtuvo mediante el análisis de porcentaje de similitud (APSIM). Además, se procedió a ejecutar un análisis de redundancia basado en distancias (ARD-bd, Legendre \& Anderson 1999) con objeto de determinar el efecto de la complejidad estructural de la pradera (densidad de pies, altura de hoja y sus desviaciones estándar respectivas) sobre la estructura de la comunidad de peces. Este análisis se complementó con una regresión múltiple multivariante, ejecutada a través de la rutina 'DISTLM' (McArdle \& Anderson 2001), sobre disimilitudes de Bray-Curtis, con propósito de conocer las variables con mayor capacidad predictiva. Se empleó la rutina 'BEST' para seleccionar el modelo con mayor parsimonia (e.g., evitando colinealidad entre variables). Las especies con una frecuencia de aparición en las muestras inferior al 5\% no fueron consideradas en los análisis multivariantes (Apéndice A). Todos los procedimientos multivariantes se ejecutaron mediante PRIMER \& PERMANOVA 6.0. (Clarke \& Gorley 2006).

\section{Resultados}

\section{ESTRUCTURA Y COMPOSICIÓN DE LA COMUNIDAD ÍCTICA}

Se registraron un total de 3.616 individuos pertenecientes a 30 especies y 15 familias (Apéndice A). Las familias más representativas, en términos de riqueza y abundancia, fueron: Sparidae con 7 especies y el $71,9 \%$ de los individuos, Labridae $(5 ; 5,23 \%)$, Syngnathidae $(3 ; 9,27 \%)$ y Mullidae $(1 ; 8,44 \%)$. Las especies más representativas en términos de abundancia y frecuencia fueron: Diplodus annularis con el $43,86 \%$ del total de individuos y $80,56 \%$ de frecuencia de aparición en las muestras, Spondyliosoma cantharus $(24,72 \%$ y $52,78 \%)$, Mullus surmuletus $(8,43 \%$ y $58,33 \%)$, Syngnathus typhle (8,3\% y 
94,44\%), Symphodus trutta (4,62\% y 47,22\%), Sparisoma cretense $(1,58 \%$ y $38,89 \%)$, Diplodus vulgaris $(1,41 \%$ y $22,22 \%)$, Pagellus erythrinus $(1,22 \%$ y $16,67 \%)$, Stephanolepis hispidus $(0,83 \%$ y $41,67 \%)$ y Sphoeroides marmoratus $(0,61 \%$ y $41,67 \%)$; estas 10 especies representan el $95,58 \%$ del total de individuos capturados. El $76,7 \%$ de las especies registradas tienen interés comercial y son objetivo de la flota artesanal canaria (Franquet \& Brito 1995). El número medio de individuos por arrastre fue 100,44 $\pm 16,9$ (media \pm error estándar, $\mathrm{n}=$ 36), con valores mínimo de 5 y máximo de 453 . El número medio de especies por arrastre fue 7,61 $\pm 0,56$, oscilando entre 3 y 15 . El 95,02\% de los individuos presentaron una longitud total inferior a $10 \mathrm{~cm}$, de los cuales la mayoría eran juveniles $(90,02 \%)$.

\section{DIFERENCIAS ENTRE ISLAS Y PRADERAS}

Los arrastres en Gran Canaria (GC) presentaron el mayor valor de riqueza ( 22 especies) y número medio de especies por muestra $(10 \pm 0,77$, media \pm error estándar, $\mathrm{n}=12$, Fig. 2A). La mayor abundancia (2.317 individuos) y número medio de individuos por muestra $(193,08 \pm 35,3)$ se registró en Lanzarote (LZ) (Fig. 2C). En GC también se registró el mayor valor medio del índice de Shannon-Wiener $(0,631$ $\pm 0,05)$, mientras que para el índice de equidad fue Fuerteventura (FV) la isla que registró el mayor valor medio $(0,693 \pm 0,06$, Fig. 2E). Estas diferencias entre islas, sin embargo, no fueron significativas para todos estos descriptores univariantes de la comunidad íctica (ANDEVA, factor 'isla', $P>0,05$ en todos los casos, Tabla 1).
Figura 2. A-B. S: riqueza ( ${ }^{\circ}$ de especies); $R$ : riqueza media $\left(n^{\circ}\right.$ de especies 100 $\mathrm{m}^{-2}$ ). C-D. Abundancia $\left(\mathrm{n}^{\circ}\right.$ de ind 100 $\mathrm{m}^{-2}$ ). E-F. $\mathrm{H}^{\prime}$ : índice de Shannon-Wiener y $\mathrm{J}$ ': índice de Pielou o equidad, para las islas ( $L Z=$ Lanzarote, $\mathrm{FV}=$ Fuerteventura, $\mathrm{GC}=$ Gran Canaria) y praderas dentro de cada isla. Se presentan los valores medios; las barras de error son el error estándar de las medias / A-B. S: richness ( $n^{\circ}$ species); $R$ : mean richness ( $n^{\circ}$ species $\left.100 \mathrm{~m}^{-2}\right)$. C-D. Abundance $\left(\mathrm{n}^{\circ}\right.$ ind $\left.100 \mathrm{~m}^{-2}\right)$. E-F. $\mathrm{H}^{\prime}$ : ShannonWiener's index and J': Pielou's or electivity index, for each island ( $L Z$ = Lanzarote, $F V=$ Fuerteventura, GC = Gran Canaria) and seagrass meadows within each island. Bars are mean values and error bars are SE of means
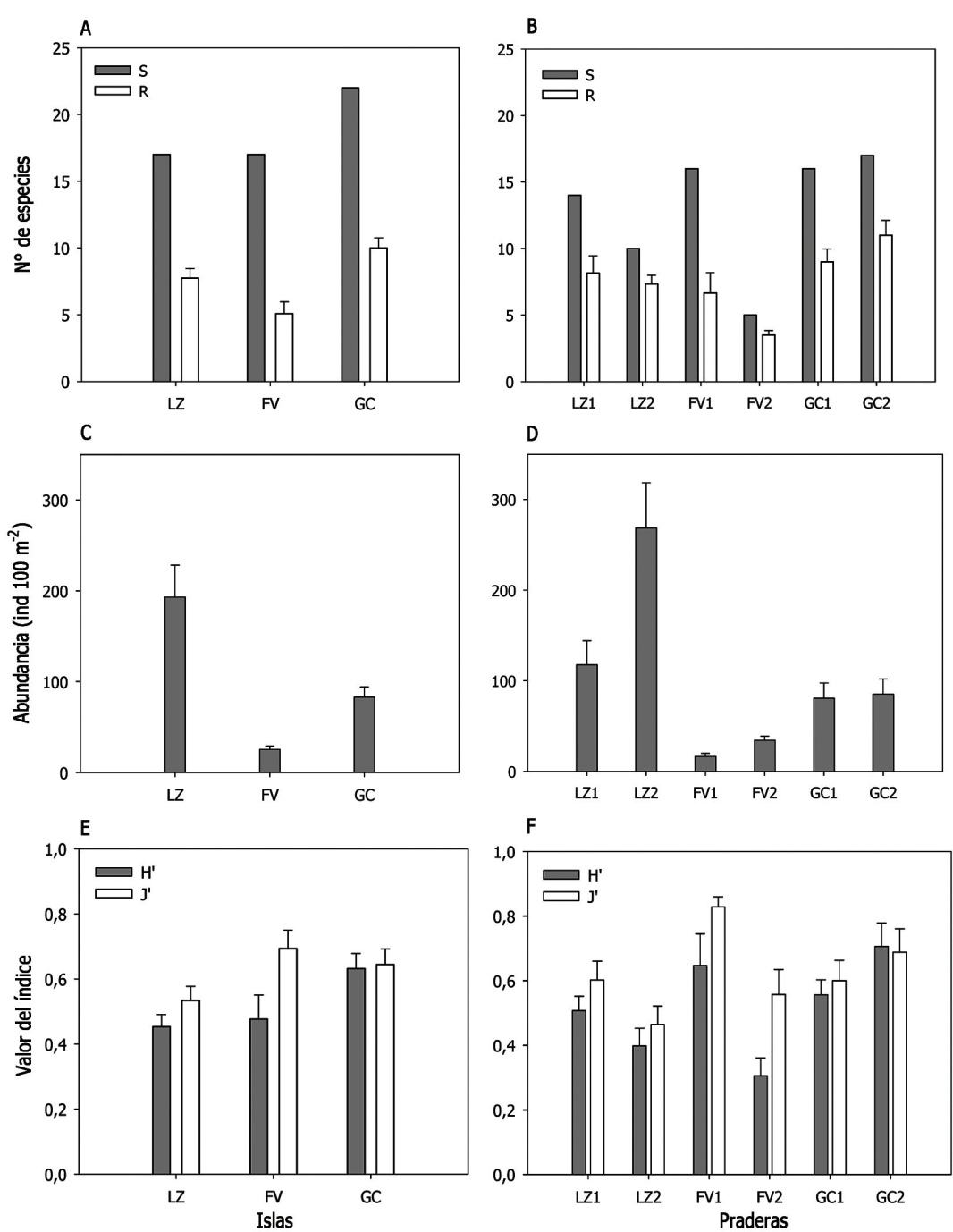
Tabla 1. Resultados de los ANDEVAs univariantes y multivariante para contrastar el efecto de la variación espacial entre islas y praderas sobre la riqueza, la abundancia total de individuos, los índice de diversidad de Shannon-Wiener (H') y equidad (J'), la abundancia total de individuos de cada categoría espacial y trófica, y la estructura de la comunidad. gl: grados de libertad; MS: cuadrados medios; $F$ : valor del estadístico; * $P<0,05,{ }^{* *} P<0,01 /$ Results of univariate and multivariate ANOVAs testing for spatial differences between islands and meadows within islands in regards to the richness, total abundance of individuals, Shannon-Wiener's ( $H^{\prime}$ ) and electivity ( $\left.J^{\prime}\right)$ index, total abundance of individuals for each spatial and trophic group, and assemblage structure. gl: degree of freedom; MS: means squares; $F$ : statistical value ${ }^{*} P<0.05,{ }^{* *} P<0.01$

\begin{tabular}{|c|c|c|c|c|c|c|c|c|c|c|c|c|c|}
\hline \multirow[t]{2}{*}{$\begin{array}{l}\text { Fuente de } \\
\text { variación }\end{array}$} & \multirow[t]{2}{*}{$\mathrm{gl}$} & \multicolumn{2}{|c|}{ Riqueza } & \multicolumn{2}{|c|}{ Abundancia } & \multicolumn{2}{|c|}{$\mathrm{H}^{\prime}$} & \multicolumn{2}{|c|}{ J' } & \multicolumn{2}{|c|}{$\begin{array}{l}\text { Estructura de la } \\
\text { comunidad }\end{array}$} & & \\
\hline & & MS & $F$ & MS & $F$ & MS & $F$ & MS & $F$ & MS & Pseudo- $F$ & & \\
\hline Isla & 2 & 2,762 & 5,269 & 206,054 & 5,669 & 0,0590 & 0,7417 & 0,032 & 0,757 & $11.267,346$ & 1,617 & & \\
\hline Pradera (Isla) & 3 & 0,524 & 2,366 & 36,343 & $5,169^{* *}$ & 0,0795 & $6,4281 * \sharp$ & 0,042 & $4,083^{*}$ & $6.964,049$ & $5,747 * *$ & & \\
\hline Residual & 30 & 0,221 & & 7,030 & & 0,0124 & & 0,010 & & $1.211,739$ & & & \\
\hline \multirow[t]{2}{*}{$\begin{array}{l}\text { Fuente de } \\
\text { variación }\end{array}$} & \multirow[t]{2}{*}{$\mathrm{gl}$} & \multicolumn{2}{|c|}{ Bentónicos } & \multicolumn{2}{|c|}{ Epibentónicos } & \multicolumn{2}{|c|}{ Macrófagos } & \multicolumn{2}{|c|}{ Mesófagos } & \multicolumn{2}{|c|}{ Micrófagos } & \multicolumn{2}{|c|}{ Omnívoros } \\
\hline & & MS & $F$ & MS & $F$ & MS & $F$ & MS & $F$ & MS & $F$ & MS & $F$ \\
\hline Isla & 2 & 45,925 & 13,312 & 169,038 & 3,233 & 0,754 & 1,745 & 20,732 & 4,228 & 3,787 & 7,816 & 231,269 & 3,864 \\
\hline Pradera (Isla) & 3 & 3,449 & $3,107 *$ & 52,274 & $7,209 * *$ & 0,432 & 0,575 & 4,902 & 2,411 & 0,484 & 1,302 & 59,853 & $5,815 * *$ \\
\hline Residual & 30 & 1,110 & & 7,251 & & 0,751 & & 2,033 & & 0,372 & & 10,292 & \\
\hline
\end{tabular}

El análisis de ordenación ACP mostró una separación de la ictiofauna de las 3 islas estudiadas, donde los poblamientos de GC y LZ fueron más similares entre sí, y se diferenciaron de los de FV (Fig. 3). Sin embargo, las diferencias en la estructura de la comunidad de ictiofauna entre islas no fueron significativas (AMDEVA, factor 'Isla', $P>0,05$, Tabla 1). De hecho, la cantidad de variabilidad explicada por el factor 'isla' $(6,64 \%$ de la variabilidad) fue considerablemente inferior a la variabilidad explicada por el factor 'pradera' $(27,28 \%$ de la variabilidad), mientras que la menor de las escalas espaciales (e.g., variabilidad entre arrastres) explicó la mayor cantidad de variabilidad $(66,07 \%)$.

La familia Sparidae presentó el mayor número de especies e individuos en las tres islas, seguida en términos de riqueza por Labridae en LZ y GC, y Syngnathidae en $\mathrm{FV}$; mientras que en términos de abundancia le siguieron Mullidae en LZ, Labridae en GC y Syngnathidae en FV. La prueba APSIM indicó que $D$. annularis, $S$. cantharus y M. surmuletus contribuyeron al $60 \%$ y $50 \%$ de la disimilitud entre LZ y FV, y LZ y GC respectivamente; mientras que las dos primeras especies, junto a $S$. trutta, contribuyeron al 40\% de disimilitud entre FV y GC (Tabla 2).

A nivel de praderas, $\mathrm{GC} 2$ registró la mayor riqueza (17 especies) y número medio de especies por muestra (11 \pm

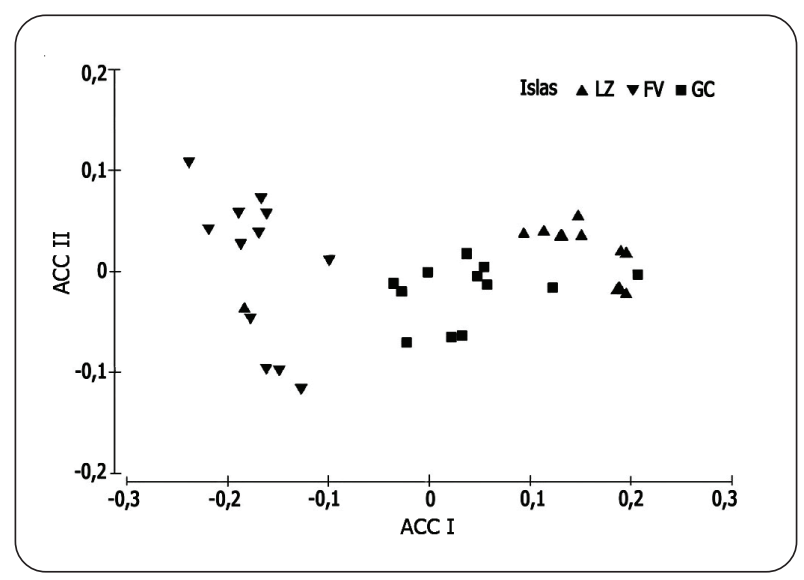

Figura 3. Resultado de la técnica de ordenación ACP mostrando las diferencias en la estructura de la comunidad íctica entre islas / Ordination plot through the CAP routine showing differences in the fish assemblage structure between islands

1,13, Fig. 2B); sin embargo, estas diferencias para la riqueza fueron no significativas (ANDEVA, 'riqueza', $P>0,05$, Tabla 1). LZ2 registró la mayor abundancia (1.611 individuos) y número medio de individuos por muestra (268,5 $\pm 49,94$, Fig. 2D); en este caso, si se detectaron diferencias significativas entre praderas (ANDEVA, 'abundancia', $P<0,01$, Tabla 1). La pradera con mayor índice de diversidad fue $\operatorname{GC2}(0,706 \pm 0,07$, Fig. 2F), 
Tabla 2. Resultados de la rutina APSIM para contrastar las disimilitudes entre las tres islas, basado en datos de abundancia. Las especies están ordenadas por orden decreciente de contribución. $\bar{\delta}_{\mathrm{i}}$, promedio de disimilitud; $\bar{\delta}_{i} \%$, contribución de cada especie al promedio de disimilitud; DE: desviación estándar / Results of the SIMPER routine testing for dissimilarities between each pair of islands, based on abundance data. The species are in decreasing order of contribution. $\bar{\delta}_{\mathrm{i}}$, mean dissimilarity; $\bar{\delta}_{\mathrm{i}} \%$, contribution of each species to mean dissimilarity; DE: standard deviation

\begin{tabular}{|c|c|c|c|c|c|c|c|c|}
\hline \multicolumn{3}{|c|}{$\begin{array}{l}\text { Lanzarote } v s \text {. Fuerteventura } \\
\qquad \bar{\delta}_{i}=77,26\end{array}$} & \multicolumn{3}{|c|}{$\begin{array}{l}\text { Lanzarote } v_{s .} \text { Gran Canaria } \\
\qquad \bar{\delta}_{i}=61,75\end{array}$} & \multicolumn{3}{|c|}{$\begin{array}{l}\text { Fuerteventura vs. Gran Canaria } \\
\qquad \bar{\delta}_{i}=69,80\end{array}$} \\
\hline Especies & $\bar{\delta}_{i} \%$ & $\bar{\delta}_{i / \mathrm{DE}}$ & Especies & $\bar{\delta}_{i} \%$ & $\bar{\delta}_{i / \mathrm{DE}}$ & Especies & $\bar{\delta}_{i} \%$ & $\bar{\delta}_{i} / \mathrm{DE}$ \\
\hline D. annularis & 22,95 & 1,31 & D. annularis & 20,23 & 1,21 & S. cantharus & 16,79 & 1,08 \\
\hline S. cantharus & 21,46 & 1,83 & S. cantharus & 15,3 & 1,33 & D. annularis & 13,79 & 1,3 \\
\hline M. surmuletus & 14,25 & 2,1 & M. surmuletus & 11,55 & 2,02 & S. trutta & 9,77 & 0,95 \\
\hline S. trutta & 5,89 & 1,89 & S. trutta & 7,59 & 1,46 & S. typhle & 6,87 & 1,17 \\
\hline S. typhle & 5,59 & 1,06 & S. cretense & 4,91 & 1,12 & S. cretense & 6,62 & 1,21 \\
\hline S. saurus & 3,16 & 0,48 & S. typhle & 4,71 & 1,02 & M. surmuletus & 4,87 & 1,27 \\
\hline P. erythrinus & 3,15 & 0,54 & D. vulgaris & 4,06 & 0,77 & D. vulgaris & 4,81 & 0,63 \\
\hline N. ophidion & 2,79 & 0,94 & S. hispidus & 3,54 & 1,53 & S. hispidus & 4,19 & 1,39 \\
\hline B. podas & 2,73 & 0,62 & S. marmoratus & 3,03 & 1,38 & S. marmoratus & 4,12 & 1,53 \\
\hline S. hispidus & 2,15 & 0,69 & S. acus & 2,81 & 1,39 & N. ophidion & 3,44 & 1,11 \\
\hline D. dentex & 1,99 & 0,92 & P. erythrinus & 2,81 & 0,52 & G. niger & 3,36 & 0,87 \\
\hline S. marmoratus & 1,67 & 0,66 & S. saurus & 2,43 & 0,54 & S. acus & 3,22 & 1,21 \\
\hline G. niger & 1,62 & 0,47 & D. dentex & 1,9 & 0,91 & B. podas & 2,24 & 0,63 \\
\hline X. novacula & 1,5 & 0,78 & G. niger & 1,78 & 0,73 & P. octolineatum & 1,89 & 0,42 \\
\hline
\end{tabular}

Tabla 3. Características estructurales de las praderas marinas. Densidad $\left(\mathrm{n}^{\circ}\right.$ de pies $\mathrm{m}^{-2}$; media $\left.\pm \mathrm{DE}\right)$; Altura (cm; media $\pm \mathrm{DE}$ ); Distribución y Superficie $\left(\mathrm{m}^{2}\right)$ de acuerdo con Espino et al. (2003) / Structural characteristics of the seagrass meadows. Density $\left(\mathrm{n}^{\circ}\right.$ of shoots $\mathrm{m}^{-2}$; mean $\left.\pm D E\right)$; Height $(\mathrm{cm}$; mean $\pm D E)$; Distribution and Surface $\left(\mathrm{m}^{2}\right)$ according to Espino et al. (2003)

\begin{tabular}{lcccr}
\hline \multicolumn{1}{c}{ Pradera } & Densidad & Altura & Distribución & Superficie \\
\hline Playa Blanca (LZ1) & $640,40 \pm 147,32$ & $22,53 \pm 4,72$ & continua & 214.588 \\
Los Pocillos (LZ2) & $910,80 \pm 106,44$ & $33,06 \pm 3,55$ & continua & 652.040 \\
Jandía (FV1) & $549,53 \pm 144,16$ & $36,78 \pm 2,74$ & parches & 147.505 \\
La Entallada (FV2) & $1.008,80 \pm 88,52$ & $36,51 \pm 6,37$ & parches & 149.268 \\
Veneguera (GC1) & $606,36 \pm 70,85$ & $44,37 \pm 2,69$ & parches & 52.663 \\
Arinaga (GC2) & $1.248,80 \pm 248,79$ & $43,20 \pm 3,73$ & parches & 261.550 \\
\hline
\end{tabular}

mientras que para el índice de equidad fue FV1 $(0,829 \pm$ 0,03 , Fig. 2F). Las diferencias entre praderas fueron significativas para ambos índices (ANDEVA, $\mathrm{H}^{\prime}, P<0,01$; ANDEVA, $\mathrm{J}^{\prime}, P<0,05$, Tabla 1). Para los grupos espaciales se registraron diferencias significativas entre praderas para la abundancia de las especies 'bentónicas' (ANDEVA, $P<0,05$, Tabla 1, Fig. 4A) y 'epibentónicas' (ANDEVA, $P<0,01$, Tabla 1, Fig. 4A). Para la abundancia de los grupos tróficos, solo fueron detectadas en el caso de los 'omnívoros' (ANDEVA, $P<0,01$, Tabla 1, Fig. 4B).

\section{EFecto de la estructura del hábitat SObre la COMUNIDAD ÍCTICA}

Las características estructurales de las 6 praderas marinas estudiadas se presentan en la Tabla 3. Las diferencias en densidad de pies $\left(F_{2,35}=0,1025, P=0,86\right)$ y altura de hojas $\left(F_{2,35}=5,3006, P=0,07\right)$ no fueron significativas entre islas; motivado por las diferencias significativas entre praderas $\left(F_{2,35}=33,2141, P=0,0002 ; F_{2,35}=7,9111, P=\right.$ 0,001 , respectivamente). El análisis de redundancia (ARDbd, Fig. 5) reveló que la densidad media de pies y la 


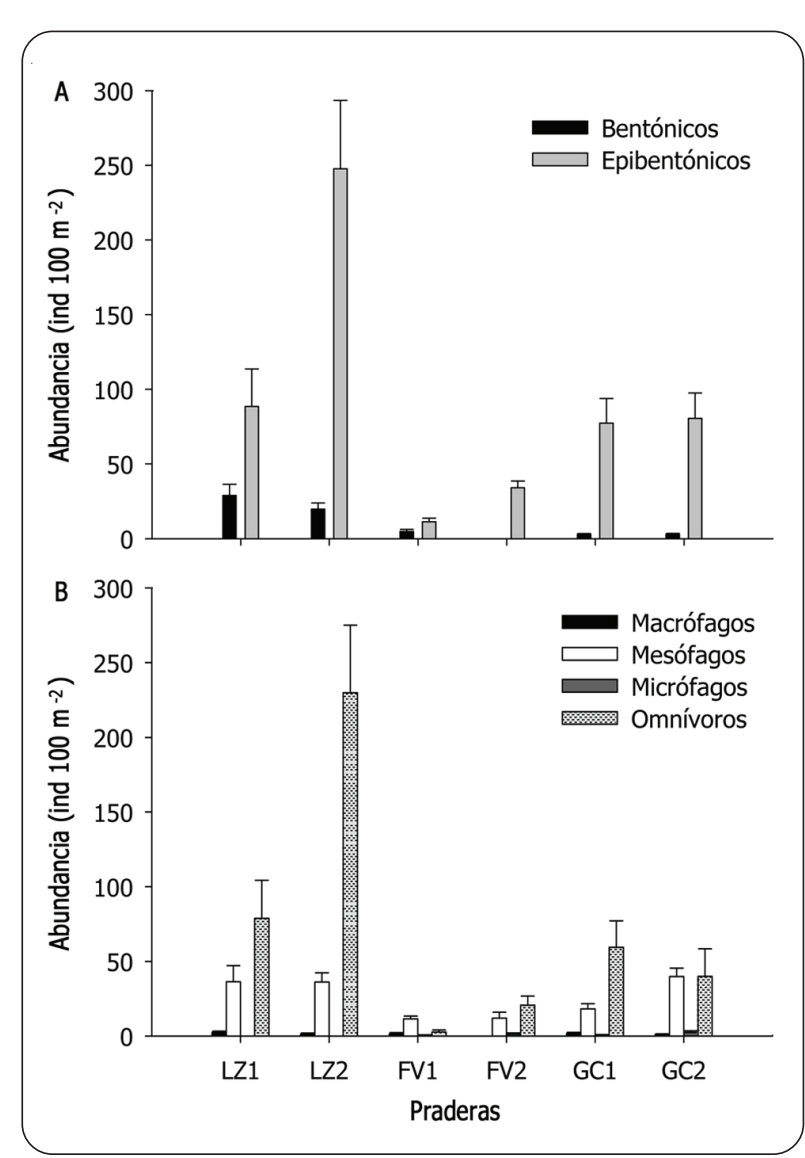

Figura 4. Abundancia de los grupos espaciales (A) y tróficos (B) en cada una de las praderas. Se presentan los valores medios; las barras de error son el error estándar de las medias / Abundance of the spatial (A) and trophic (B) groups in each seagrass meadows. Bars are mean values and error bars are standard error of means

variabilidad en la longitud de hojas (e.g., desviación estándar de la altura) fueron, de las variables estructurales estudiadas, las que más afectaron a la estructura de la comunidad íctica. El análisis de regresión múltiple mostró que la densidad media de pies, la altura media de hojas y su variación, contribuyeron significativamente $(P<0,05$, Tabla 4) a explicar aproximadamente el $32 \%$ de la variabilidad total en la estructura de la comunidad íctica. La densidad media de pies y la variabilidad de la altura de hojas fueron las que más contribuyeron a explicar la variabilidad en la estructura de la comunidad $(\sim 22 \%)$.

\section{Discusión}

\section{VARIABILIDAD ESPACIAL ENTRE ISLAS Y PRADERAS}

Los resultados de este estudio indicaron que la ictiofauna asociada a las praderas de Cymodocea nodosa en las

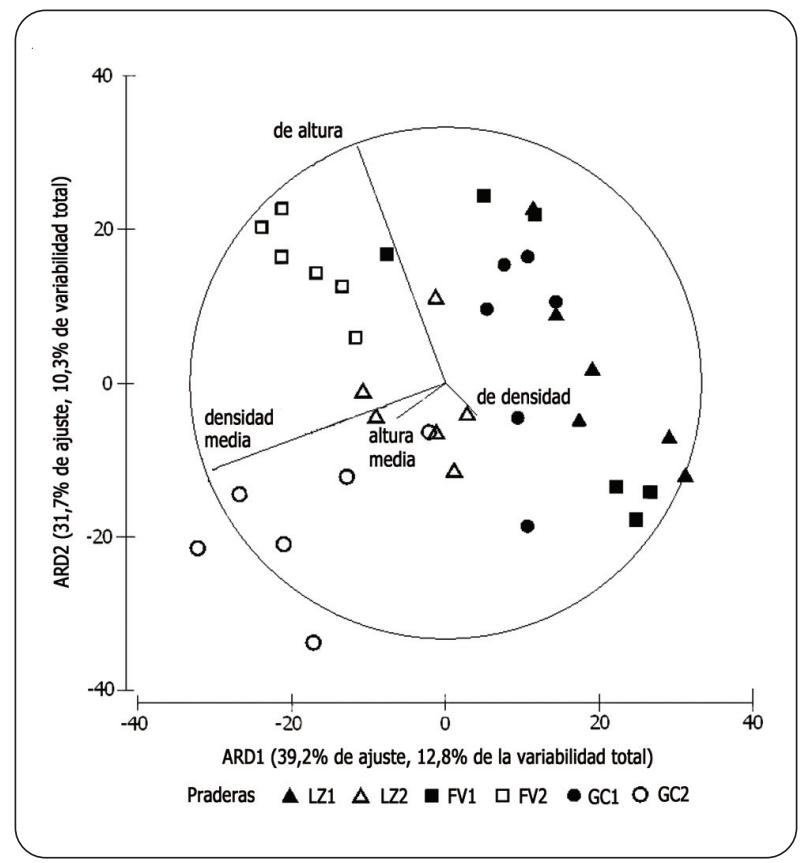

Figura 5. Resultado de la prueba ARD-bd mostrando el efecto de la complejidad estructural de las praderas sobre las diferencias en la estructura de la comunidad íctica entre islas (de = desviación estándar) / Result of the db-RDA analysis showing the effect of the structural complexity of the seagrass meadows over the differences in the fish assemblage structure between islands (de $=$ standard deviation $)$

islas Canarias presentó mayor heterogeneidad espacial entre praderas que entre islas: es decir, a una escala espacial intermedia. A pesar de que la ordenación ACP mostró una relativa separación entre la ictiofauna de las tres islas, los contrastes multivariante (AMDEVA) y univariantes (ANDEVA sobre la riqueza, abundancia y diversidad de la comunidad) han demostrado que no hay diferencias significativas en la estructura de la comunidad y en los descriptores univariantes del poblamiento íctico a la mayor de las escalas espaciales: entre islas. En cualquier caso, los análisis univariantes de ciertos descriptores (riqueza, abundancia, grupos micrófagos y omnívoros) mostraron valores cercanos al límite de rechazo de la hipótesis nula ( $P=0,06$ en los 4 casos); esto sugiere que las diferencias en la variabilidad espacial encontrada entre islas para estos descriptores, aunque no se hicieron patentes, quedaron enmascaradas por causa de la alta variabilidad detectada a nivel de praderas. La mayor variabilidad en la estructura de la comunidad se detectó entre arrastres individuales y podría explicarse, principalmente, porque: i) las especies de peces más abundantes presentaron una distribución agregada (ver Anexo 1) y ii) la distancia entre los arrastres fue pequeña 
Tabla 4. Resultado de la regresión múltiple multivariante contrastando el efecto de las variables estructurales de praderas de Cymodocea nodosa sobre la estructura de la comunidad de peces. Se incluye la proporción de variabilidad total explicada por cada variable y la acumulada al incluir sucesivas variables en el modelo / Results of the multivariate multiple regressions testing the effect of the structural variables of Cymodocea nodosa seagrass meadows on the fish assemblage structure. The proportion of the explained variability by each variable is included, as well as the accumulated proportion when successive variables are included in the model

\begin{tabular}{lcccc}
\hline \multicolumn{1}{c}{ Variables } & Pseudo- $F$ & $P$ & $\begin{array}{c}\text { Proporción de la } \\
\text { variabilidad explicada }\end{array}$ & $\begin{array}{c}\text { Variabilidad } \\
\text { total acumulada }\end{array}$ \\
\hline Densidad media de pies & 4,598 & 0,0008 & 0,119 & 0,119 \\
DE Altura de hoja & 4,078 & 0,0018 & 0,096 & 0,216 \\
Altura media de hoja & 2,913 & 0,0136 & 0,065 & 0,281 \\
DE Densidad de pies & 1,951 & 0,0838 & 0,042 & 0,324 \\
\hline
\end{tabular}

$\mathrm{DE}=$ desviación estándar

en relación con el alto rango de movilidad de varias de las especies, como ha sucedido en estudios de variabilidad espacial de peces en arrecifes rocosos templados (Anderson \& Millar 2004, Ordines et al. 2005, Tuya et al. 2011) y en praderas de fanerógamas marinas, como es el caso de Posidonia oceanica (Moranta et al. 2006).

Las diferencias detectadas entre praderas, tanto en la estructura de la comunidad (análisis multivariante), como para determinados atributos de la comunidad: abundancia, diversidad, equidad, y abundancia de los grupos bentónicos, epibentónicos y omnívoros (análisis univariantes) se deben, principalmente, a diferencias en los patrones de abundancia de individuos pertenecientes a las familias Sparidae (Diplodus annularis y Spondyliosoma cantharus), Mullidae (Mullus surmuletus) y Labridae (Symphodus trutta). Para las tres primeras especies, estas abundancias se debieron a cohortes de individuos juveniles, que están relacionadas con procesos de reclutamiento en las praderas de $C$. nodosa (Mena et al. 1993, Guidetti \& Bussotti 2000, Ribeiro et al. 2006, Verdiell-Cubedo et al. 2007, Espino et al. 2011). Además, las mayores abundancias de individuos se registraron en las praderas con mayor superficie (LZ1, LZ2 y GC2) y continuidad $(\sim 100 \%$ de cobertura), confirmando que, al igual que sucede en praderas de P. oceanica en el Mediterráneo (VegaFernández et al. 2005, Moranta et al. 2006), las praderas de $C$. nodosa con mayor tamaño en Canarias favorecen la abundancia de individuos de tallas pequeñas. La variabilidad en la abundancia de peces entre praderas marinas observada en el Mediterráneo (Moranta et al.
2006), también registró diferencias significativas y estuvo relacionada con altas densidades de individuos pequeños (e.g., D. annularis), probablemente asociados a procesos de reclutamiento.

Las diferencias observadas para el índice de diversidad se deben a dos praderas: GC2 y FV1. En GC2, se registró el mayor valor medio de este descriptor. La coexistencia de hábitats de arena y pradera, y los límites entre ellos podrían explicar el mayor número de especies presentes, como sucede en otras praderas fragmentadas del Mediterráneo (Vega-Fernández et al. 2005). Esta mayor variedad de hábitats disponibles, incluidos los corredores de arena, favorecería la presencia de especies como Xyrichtys novacula y Mullus surmuletus. Por otro lado, la cercanía de esta pradera $(10 \mathrm{~m})$ a otros tipos de hábitats como arrecifes rocosos con comunidades de macroalgas, facilitarían la transición de especies como Parapristipoma octolineatum, Thalassoma pavo, Scorpaena porcus, Sparisoma cretense y Symphodus spp, características de fondos rocosos (Brito et al. 2002, Tuya et al. 2004). En el caso de FV1, dos de los arrastres se realizaron muy cerca de un emisario submarino de aguas residuales, donde la entrada de materia orgánica y la perturbación ticmotrópica producen un alto valor de riqueza y biomasa íctica en comparación con zonas adyacentes (Tuya et al. 2002a), lo que explicaría el mayor número de especies detectadas en esta zona de la pradera.

En contraste con nuestros resultados, los estudios sobre variabilidad espacial de la ictiofauna asociada a praderas de Posidonia oceanica del NW del Mediterráneo han mostrado que la mayor variabilidad espacial se 
detectó a la mayor escala espacial (diferencias entre localidades separadas por $>100 \mathrm{~km}$ ). Por el contrario, las diferencias entre praderas $(<10 \mathrm{~km})$ no fueron significativas; mientras que a la menor escala, la variabilidad asociada a los arrastres $(<1 \mathrm{~km})$, fue también importante (Moranta et al. 2006). Las diferencias entre ambos estudios podrían tener varias explicaciones. En primer lugar, los ecosistemas litorales de las islas Canarias, incluidas las praderas de C. nodosa, se caracterizan por su pequeño tamaño (Aguilera et al. 1994), al tratarse de islas oceánicas con escasa plataforma costera: lo que derivaría a un mayor aislamiento entre praderas y una mayor heterogeneidad ambiental y estructural entre las mismas, sobre todo en comparación con ecosistemas litorales continentales. En segundo lugar, por la distinta distancia entre las praderas estudiadas en ambos casos: $>10 \mathrm{~km}$ en nuestro estudio, frente $\mathrm{a}<10 \mathrm{~km}$ en el caso del Mediterráneo. En tercer lugar, porque en el estudio de Moranta et al. (2006) se compararon localidades insulares con localidades continentales, mientras que nosotros hemos estudiado praderas en tres islas oceánicas. De hecho, Barberá et al. (2005) han demostrado la existencia de una fuerte heterogeneidad estructural a pequeña (sitios separados por $100 \mathrm{~m}$ dentro de praderas) y mediana escala (praderas separadas por $10 \mathrm{~km}$ dentro de islas) en contraste con la ausencia de variabilidad estructural en las praderas entre islas (separadas por $100 \mathrm{~km}$ ).

El grupo de las especies epibentónicas fue el más abundante en todas las praderas, siendo las abundancias de Diplodus annularis y Spondyliosoma cantharus en LZ1 y LZ2 las que explicaron las diferencias consistentes detectadas, mientras que la abundancia de M. surmuletus en estas mismas praderas determinó las diferencias para el grupo bentónico. En el caso de las categorías tróficas, las diferencias encontradas se deben a los omnívoros $(D$. annularis, $S$. cantharus), que fueron los más abundantes en todas las praderas, excepto en GC2 donde los mesófagos (Symphodus trutta) registraron una abundancia similar.

\section{EFecto de la estructura del hábitat sobre la COMUNIDAD ÍCTICA}

En general, las praderas de fanerógamas marinas con mayor complejidad estructural albergan ictiofaunas más ricas y diversas (Heck \& Orth 1980, Bell \& Westoby 1986, Bell \& Pollard 1989, Jenkins \& Wheatley 1998, Gray et al. 1998, Guidetti \& Bussotti 2000). En nuestro estudio, la densidad media de pies ( $12 \%$ de la variabilidad) y la variación de la altura del estrato foliar $(9 \%$ de la variabilidad) fueron los parámetros que más influyeron en la estructura de la comunidad íctica. En estudios previos, la influencia de la densidad y la altura de las plantas sobre la estructura de la ictiofauna han sido demostradas a escala espacial pequeña (Bell \& Westoby 1986). Más recientemente y en concordancia con nuestros resultados, Gullström et al. (2008) han demostrado que praderas con mayor heterogeneidad en el estrato foliar constituyen un hábitat más adecuado para los individuos juveniles de peces, mientras que Hori et al. (2009) han descrito una correlación positiva entre la altura de la bóveda de la pradera y la ictiofauna juvenil asociada. Sin embargo, es necesario señalar que, a escalas espaciales medias y grandes, existen otros factores que pueden influenciar las características de esta ictiofauna (Moranta et al. 2006), tales como la variedad de ecosistemas adyacentes (Dorenbosch et al. 2006), su distancia a las praderas (Jelbart et al. 2007), la presión pesquera (GarcíaRubies \& Zabala 1990), procesos de reclutamiento (GarcíaRubies \& Macpherson 1995), competición o depredación (Heck \& Orth 1980) o las propias características hidrológicas (Letourneur et al. 2003).

En conclusión, la ictiofauna asociada a las praderas de Cymodocea nodosa en las islas Canarias mostró mayor heterogeneidad espacial a nivel de praderas que de islas. Las diferencias observadas se deben, principalmente, a variaciones en la abundancia de determinadas especies de peces más que a la riqueza y composición de la comunidad, y están relacionadas con procesos de reclutamiento. Se confirma el papel que juegan estas praderas marinas como hábitat de guardería, especialmente aquellas con una mayor complejidad estructural. No obstante, la generalización de los resultados de este estudio es limitada, debido a la falta de replicación temporal en el diseño experimental. Futuros estudios deberían abordar el análisis de la variabilidad espacial integrando los cambios diarios y estacionales que se producen en la comunidad íctica. La incorporación de un mayor número de muestras considerando otras praderas permitiría desarrollar un macroanálisis mejor de este ecosistema.

\section{Agradecimientos}

Queremos agradecer el apoyo económico de la Viceconsejería de Medio Ambiente (Consejería de Medio Ambiente y Ordenación Territorial del Gobierno de Canarias) en el desarrollo del Programa de Seguimiento de Especies Amenazadas (SEGA Cymodocea nodosa 2003). Agradecemos la colaboración, en diversos aspectos 
de este trabajo, de los Drs. Mateo Garrido Sanahuja y Rogelio Herrera Pérez (Consejería de Medio Ambiente y Ordenación Territorial) y a D. Oscar Tavío Cruz (Agencia de Protección del Medio Urbano y Natural). Gracias también a tres revisores anónimos por sus valiosos comentarios sobre el manuscrito.

\section{LiTERATURA CITADA}

Aguilera F, A Brito, C Castilla, A Díaz, JM FernándezPalacios, A Rodríguez, F Sabaté \& J Sánchez. 1994. Canarias. Economía, ecología y medio ambiente, 361 pp. Francisco Lemus Editor, La Laguna.

Anderson MJ. 2001. A new method for non-parametric multivariate analysis of variance. Austral Ecology 26: 32-46.

Anderson MJ \& TJ Willis. 2003. Canonical analysis of principal coordinates: A useful method of constrained ordination for ecology. Ecology 84: 511-525.

Anderson MJ \& RB Millar. 2004. Spatial variation and effects of habitat on temperate reef fish assemblages in northeastern New Zealand. Journal of Experimental Marine Biology and Ecology 305: 191-221.

Barberá C, F Tuya, A Boyra, P Sánchez-Jerez, I Blanch \& RJ Haroun. 2005. Spatial variation in the structural parameters of Cymodocea nodosa seagrass meadows in the Canary Islands: a multiscaled approach. Botánica Marina 48: 122-126.

Beck MW, KL Heck Jr, KW Able, DL Childers, DB Eggleston, BM Gillanders, B Halpern, CG Hays, K Hoshino, TJ Minello, RJ Orth, PF Sheridan \& MP Weinstein. 2001. The identification, conservation, and management of estuarine and marine nurseries for fish and invertebrates. BioScience 51: 633-641.

Bell JD \& ML Harmelin-Vivien. 1983. Fish fauna of french Mediterranean Posidonia oceanica seagrass meadows. 2. Feeding habits. Tethys 11: 1-14.

Bell JD \& DA Pollard. 1989. Ecology of fish assemblages and fisheries associated with seagrasses. En: Larkum AWD, AJ McComb \& SA Shepherd (eds). Biology of seagrasses: a treatise on the biology of seagrasses with special reference to the Australian region, pp. 565-609. Elsevier, New York.

Bell JD \& M Westoby. 1986. Importance of local changes in leaf height and density to fish and decapods associated with seagrasses. Journal of Experimental Marine Biology and Ecology 104: 249-274.

Bonaca MO \& L Lipej. 2005. Factors affecting habitat occupancy of fish assemblage in the Gulf of Trieste (Northern Adriatic Sea). Marine Ecology 26: 42-53.

Brito A, PJ Pascual, JM Falcón, A Sancho \& G González. 2002. Peces de las islas Canarias. Catálogo comentado e ilustrado, 419 pp. Francisco Lemus Editor, La Laguna.

Clarke KR \& RN Gorley. 2006. PRIMER v6: User Manual/ Tutorial, 192 pp. PRIMER-E, Plymouth.
Costa MJ, CI Santos \& HN Cabral. 2002. Comparative analysis of a temperate and a tropical seagrass bed fish assemblages in two estuarine systems: The Mira estuary (Portugal) and the Mussulo Lagoon (Angola). Cahiers de Biologie Marine 43: 73-81.

Deudero S, JK Pinnegar, NVC Polunin, G Morey \& B Morales-Nin. 2004. Spatial variation and ontogenetic shifts in the isotopic composition of Mediterranean littoral fishes. Marine Biology 145: 971-981.

Dorenbosch M, MGG Grol, I Nagelkerken \& G Van der Velde. 2006. Different surrounding landscape may result in different fish assemblages in East African seagrass beds. Hydrobiologia 563: 45-60.

Dulcic J, M Kraljevic, B Grbec \& A Pallaoro. 1997. Composition and temporal fluctuations of inshore juvenile fish populations in the Kornati Archipelago, eastern middle Adriatic. Marine Biology 129: 267-277.

Elliott M \& F Dewailly. 1995. The structure and components of European estuarine fish assemblages. Netherlands Journal of Aquatic Ecology 29: 397-417.

Espino F. 2004. Una metodología para el estudio de las fanerógamas marinas en Canarias. Revista de la Academia Canarias de Ciencias XV: 237-256.

Espino F, M Garrido, R Herrera \& O Tavío. 2003. Seguimiento de especies amenazadas (Programa SEGA). Cymodocea nodosa. Lanzarote, Fuerteventura y Gran Canaria. Informe Técnico de la Viceconsejería de Medio Ambiente, Tomos I, II y III. 227 pp. Gobierno de Canarias, Las Palmas de Gran Canaria.

Espino F, F Tuya, I Blanch \& RJ Haroun. 2008. Los Sebadales en Canarias. Oasis de vida en los fondos arenosos, 68 pp. BIOGES. Universidad de Las Palmas de Gran Canaria, Las Palmas de Gran Canaria.

Espino F, F Tuya, A Brito \& RJ Haroun. 2011. Ichthyofauna associated with Cymodocea nodosa meadows in the Canarian Archipelago (central eastern Atlantic): Community structure and nursery function. Ciencias Marinas 37: 157174.

Ferrell DJ, SE McNeill, DG Worthington \& JD Bell. 1993. Temporal and spatial variation in the abundance of fish associated with the seagrass Posidonia australis in southeastern Australia. Australian Journal of Marine and Freshwater Research 44: 881-899.

Franquet F \& A Brito. 1995. Especies de interés pesquero de Canarias, 143 pp. Consejería de Pesca y Transportes del Gobierno de Canarias, Santa Cruz de Tenerife.

García-Rubies A \& E Macpherson. 1995. Substrate use and temporal pattern of recruitment in juvenile fishes of the Mediterranean littoral. Marine Biology 124: 35-42.

García-Rubies A \& M Zabala. 1990. Effects of total fishing prohibition on the rocky fish assemblages of Medes Islands marine reserve (NW Mediterranean). Scientia Marina 54: 317-328. 
Gillanders BM. 2006. Seagrasses, fish, and fisheries. En: Larkum AWD, RJ Orth \& CM Duarte (eds). Seagrasses: biology, ecology and conservation pp. 503-536. Springer, Dordrecht.

Gray CA, RC Chick \& DJ McElligott. 1998. Diel changes in assemblages of fishes associated with shallow seagrass and bare sand. Estuarine, Coastal and Shelf Science 46: 849859.

Guest MA, RM Connolly \& NR Loneragan. 2003. Seine nets and beam trawls compared by day and night for sampling fish and crustacean in shallow seagrass habitats. Fisheries Research 64: 185-196.

Guidetti P \& S Bussotti. 2000. Fish fauna of a mixed meadow composed by the seagrasses Cymodocea nodosa and Zostera noltii in the Western Mediterranean. Oceanologica Acta 23: 759-770.

Guidetti P \& S Bussotti. 2002. Effects of seagrass canopy removal on fish in shallow Mediterranean seagrass (Cymodocea nodosa and Zostera noltii) meadows: a localscale approach. Marine Biology 140: 445-453.

Gullström M, M Bodin, PG Nilsson \& MC Öhman. 2008. Seagrass structural complexity and landscape configuration as determinants of tropical fish assemblage composition. Marine Ecology Progress Series 363: 241-255.

Heck KLJ \& RJ Orth. 1980. Seagrass habitats: the roles of habitat complexity, competition and predation in structuring associated fish and motile macroinvertebrate assemblages. En: Kennedy VS (ed). Estuarine perspectives, pp. 449464. Academic Press, New York.

Heck KLJ \& KA Wilson. 1987. Predation rates on decapod crustaceans in latitudinally separated seagrass communities: A study of spatial and temporal variation using tethering techniques. Journal of Experimental Marine Biology and Ecology 107: 87-100.

Hemminga MA \& CM Duarte. 2000. Seagrass ecology, 298 pp. Cambridge University Press, Cambridge.

Hori M, T Suzuki, Y Monthum, T Srisombat, Y Tanaka, M Nakaoka \& H Mukai. 2009. High seagrass diversity and canopy-height increase associated fish diversity and abundance. Marine Biology 156: 1447-1458.

Jelbart JE, PM Ross \& RM Connolly. 2007. Fish assemblages in seagrass beds are influenced by the proximity of mangrove forest. Marine Biology 150: 993-1002.

Jenkins GP \& MJ Wheatley. 1998. The influence of habitat structure on nearshore fish assemblages in a southern Australian embayment: comparison of shallow seagrass, reef-algal and unvegetated sand habitats, with emphasis on their importance to recruitment. Journal of Experimental Marine Biology and Ecology 221: 147-172.

Jennings S, O Reñones, B Morales-Nin, NVC Polunin, J Moranta \& J Coll. 1997. Spatial variation in the ${ }^{15} \mathrm{~N}$ and ${ }^{13} \mathrm{C}$ stable isotope composition of plants, invertebrates and fishes on Mediterranean reefs: implications for the study of trophic pathways. Marine Ecology Progress Series 146: 109-116.
Legendre P \& MJ Anderson. 1999. Distance-based redundancy analysis: testing multispecies responses in multifactorial ecological experiments. Ecological Monographs 69: 1-24.

Letourneur Y, S Ruitton \& S Sartoretto. 2003. Environmental and benthic habitat factors structuring the spatial distribution of a summer infralittoral fish assemblage in the north-western Mediterranean Sea. Journal of the Marine Biological Association of the United Kingdom 83: 193-204.

Ludwig JA \& JF Reynolds. 1988. Statistical ecology: a primer on methods and computing, 337 pp. John Wiley \& Sons, New York.

Macpherson E, A Gordoa \& A García-Rubies. 2002. Biomass size spectra in littoral fishes in protected and unprotected areas in the NW Mediterranean. Estuarine, Coastal and Shelf Science 55: 777-788.

Manent P \& J Abella. 2005. Catálogo preliminar de la ictiofauna asociada a diferentes hábitats de una bahía somera y protegida en la reserva marina del norte de Menorca, Mediterráneo occidental. Boletín de la Sociedad de Historia Natural de Baleares 48: 87-94.

McArdle BH \& MJ Anderson. 2001. Fitting multivariate models to community data: A comment on distance-based redundancy analysis. Ecology 82: 290-297.

Mena J, JM Falcón, A Brito, FM Rodríguez \& M Mata. 1993. Catálogo preliminar de la ictiofauna de las praderas de fanerógamas marinas de la isla de Tenerife, islas Canarias. Publicaciones Especiales, Instituto Español de Oceanografía 11: 217-222.

Moranta J, M Palmer, G Morey, A Ruiz \& B Morales-Nin. 2006. Multi-scale spatial variability in fish assemblages associated with Posidonia oceanica meadows in the Western Mediterranean Sea. Estuarine, Coastal and Shelf Science 68: 579-592.

Navarro-Pérez E \& ED Barton. 2001. Seasonal and interannual variability of the Canary Current. Scientia Marina 65: 205213.

Ordines F, J Moranta, M Palmer, A Lerycke, A Suau, B Morales-Nin \& AM Grau. 2005. Variations in a shallow rocky reef fish community at different spatial scales in the western Mediterranean Sea. Marine Ecology Progress Series 304: 221-233.

Orth RJ, TJB Carruthers, WC Dennison, CM Duarte, JW Fourqurean, KL Heck Jr, AR Hughes, GA Kendrick, WJ Kenworthy, S Olyarnik, FT Short, M Waycott \& SL Williams. 2006. A global crisis for seagrass ecosystems. BioScience 56: 987-996.

Pavón-Salas N, R Herrera, A Hernández-Guerra \& R Haroun. 2000. Distributional pattern of seagrasses in the Canary islands (Central-East Atlantic Ocean). Journal of Coastal Research 16: 329-335.

Polifrone M, A Abelardo-González \& A Quevedo-Díaz. 2006. Fish community and grazing pressure on meadows of Cymodocea nodosa (Ucria) Ascherson in Gran Canaria (Canary Islands, Spain). Biologia Marina Mediterranea 13: 167-169. 
Pollard DA. 1984. A review of ecological studies on seagrassfish communities, with particular references to recent studies in Australia. Aquatic Botany 18: 3-42.

Reina-Hervás JA \& P Serrano. 1987. Structural and seasonal variations of inshore fish populations in Málaga Bay, Southeastern Spain. Marine Biology 95: 501-508.

Relini G, M Relini \& G Torchia. 2000. Fish population changes following the invasion of the allochthonous alga Caulerpa taxifolia in the Ligurian Sea (NW Mediterranean). ICES Documents, CM 2000/U: 1-17.

Reyes J, M Sansón \& J Afonso-Carrillo. 1995a. Distribution and reproductive phenology of the seagrass Cymodocea nodosa (Ucria) Ascherson in the Canary Islands. Aquatic Botany 50: 171-180.

Reyes J, M Sansón \& J Afonso-Carrillo. 1995b. Leaf phenology, growth and production of the seagrass Cymodocea nodosa at El Médano (south of Tenerife, Canary Islands). Botánica Marina 38: 457-465.

Ribeiro J, L Bentes, R Coelho, JMS Gonçalves, PG Lino, P Monteiro \& K Erzini. 2006. Seasonal, tidal and diurnal changes in fish assemblages in the Ria Formosa lagoon (Portugal). Estuarine, Coastal and Shelf Science 67: 461474.

Rozas LP \& TJ Minello. 1997. Estimating densities of small fishes and decapods crustaceans in shallow estuarine habitats: a review of sampling design with focus on gear selection. Estuaries 20: 199-213.

Tuya F, S Alvarez, GM Reuss \& A Luque. 2002a. Macrobenthic and fish communities associated with a sewage pipeline in Gran Canaria (Canary Islands). Biologia Marina Mediterranea 9: 1-10.

Tuya F, JA Martín \& A Luque. 2002b. Impact of a marina construction on a seagrass bed at Lanzarote (Canary Islands). Journal of Coastal Conservation 8: 157-162.

Tuya F, A Boyra, P Sánchez-Jerez, C Barberá \& R Haroun. 2004. Relationships between rocky-reef fish assemblages, the sea urchin Diadema antillarum and macroalgae throughout the Canarian Archipelago. Marine Ecology Progress Series 278: 157-169.
Tuya F, A Boyra, P Sánchez-Jerez \& RJ Haroun. 2005. Multivariate analysis of the bentho-demersal ichthyofauna along soft bottoms of the Eastern Atlantic: comparison between unvegetated substrates, seagrass meadows and sandy bottoms beneath sea-cage fish farms. Marine Biology 147: $1229-1237$

Tuya F, JA Martín \& A Luque. 2006. Seasonal cycle of a Cymodocea nodosa seagrass meadow and of the associated ichthyofauna at Playa Dorada (Lanzarote, Canary Islands, eastern Atlantic). Ciencias Marinas 32: 695-704.

Tuya F, T Wernberg \& MS Thomsen. 2011. The relative influence of local to regional drivers of variation in reef fishes. Journal of Fish Biology 79: 217-234.

Vega-Fernández T, M Milazzo, F Badalamenti \& G D’Anna. 2005. Comparison of the fish assemblages associated with Posidonia oceanica after the partial loss and consequent fragmentation of the meadow. Estuarine, Coastal and Shelf Science 65: 645-653.

Verdiell-Cubedo D, FJ Oliva-Paterna \& M Torralva-Forero. 2007. Fish assemblages associated with Cymodocea nodosa and Caulerpa prolifera meadows in the shallow areas of the Mar Menor coastal lagoon. Limnetica 26: 341-350.

Waycott M, CM Duarte, TJB Carruthers, RJ Orth, WC Dennison, S Olyarnik, A Calladine, JW Fourqurean, KL Heck Jr, AR Hughes, GA Kendrick, WJ Kenworthy, FT Short \& SL Williams. 2009. Accelerating loss of seagrass across the globe threatens coastal ecosystems. Proceedings of the National Academy of Sciences 106: 12377-12381.

Weinstein MP \& KL Heck. 1979. Ichthyofauna of seagrass meadows along the Caribbean coast of Panama and in the Gulf of Mexico: Composition, structure and community ecology. Marine Biology 50: 97-107.

Worthington DG, DJ Ferrell, SE McNeill \& JD Bell. 1992. Effects of the shoot density of seagrass on fish and decapods: Are correlation evident over a large spatial scales? Marine Biology 112: 139-146.

Wyda JC, LA Deegan, JE Hughes \& MJ Weaver. 2002. The response of fishes to submerged aquatic vegetation complexity in two ecoregions of the mid-Atlantic bight: Buzzards Bay and Chesapeake Bay. Estuaries 25: 86-100.

Recibido el 22 de febrero y aceptado el 08 de noviembre de 2011 